\title{
Optical characterization of two-dimensional photonic crystal cavities with indium arsenide quantum dot emitters
}

\author{
Tomoyuki Yoshie and Axel Scherer ${ }^{\mathrm{a})}$ \\ Department of Electrical Engineering, California Institute of Technology, Pasadena, California 91125
}

Hao Chen, Diana Huffaker, and Dennis Deppe

Microelectronics Research Center, Department of Electrical and Computer Engineering, The University of Texas at Austin, Austin, Texas 78712-1084

(Received 19 February 2001; accepted for publication 16 April 2001)

\begin{abstract}
We have characterized the modes within two-dimensional photonic crystal nanocavities with self-organized indium arsenide quantum dots as an active material. Highly localized donor mode resonances with 3 to $5 \mathrm{~nm}$ linewidth were observed when spatially selective optical pumping the cavities. These modes could be lithographically tuned from 1100 to $1300 \mathrm{~nm}$. Other, more extended modes, were also characterized and exhibited narrower resonance linewidths ranging from 0.6 to 2 nm. (C) 2001 American Institute of Physics. [DOI: 10.1063/1.1377851]
\end{abstract}

High quality factor $(Q)$ optical microcavities exhibit many interesting and potentially useful applications since they can be used to confine light within very small volumes. The idea of controlling spontaneous emission within such cavities originates from Purcell's pioneering work $^{1}$ on the modification of spontaneous emission in microwave cavities. Over 50 years after this initial work on trapped atoms in microwave cavities, solid state microcavities can now also be designed and constructed for the optical frequency range. So far, the geometries used for the construction of optical nanocavities include micropillars, ${ }^{2,3}$ microdisks, ${ }^{4} \mathrm{AlO}_{x}$-apertured vertical cavities, ${ }^{5}$ and photonic band gap cavities. ${ }^{6}$ All of these have been shown to be capable of significantly modifying the spontaneous emission lifetime, rate, and directionality. Among the presently available solid state microcavity geometries, photonic crystal nanocavities have attracted much attention since Meade et al. ${ }^{7}$ introduced the idea of high- $Q$ localized modes in photonic crystal slabs, and such cavities were later predicted to offer the smallest mode volumes at surprisingly large $Q$ values. ${ }^{8}$ By shrinking the cavity size until all higher-order modes are cut off, only a few modes are allowed to exist in a photonic band gap resonator cavity. So far, photonic crystal lasers have been demonstrated in both the InGaAsP/InP and InGaAs/GaAs quantum well material systems, emitting in the $1.5 \mu \mathrm{m}$ telecommunication wavelength range ${ }^{9,10}$ and at $930 \mathrm{~nm} .{ }^{11}$ However, when using quantum well material, this geometry suffers from significant reabsorption of light in the lithographically defined mirrors, since both the active light-emitting region in the cavity as well as the photonic crystal mirrors absorb light. Nonetheless, we find that two-dimensional (2D) photonic crystal slab defect cavities can be easily fabricated by using planar lithography and etching technologies and offer desired high $Q$ 's and small mode volumes.

Quantum dots (QDs) are three-dimensionally confined electronic structures with lateral dimensions on the order of the de Broglie wavelength. When used as light sources, QDs

${ }^{a)}$ Electronic mail: etcher@cco.caltech.edu provide some fascinating properties such as atom-like density of states and a narrow emission linewidth. Moreover, the past efforts on growth of QDs, ${ }^{12,13}$ especially self-assembled QDs, now enable us to use QDs as reliable emission sources. Here, we show that active QDs can be embedded in high- $Q$ photonic crystal microcavities to create devices with very interesting characteristics. For solid state microcavities, an ensemble of QDs or a single QD could be used as a narrow spectrum light source which matches the linewidth of a high finesse cavity. It is predicted that such a QD source, included in an optical nanocavity, would result in a very low threshold laser source. The $\operatorname{In}(\mathrm{Ga})$ As system is also found to have low surface recombination rate, ${ }^{14}$ and therefore is a good candidate for constructing lasers from photonic crystal nanocavities in which large surface to volume ratios are unavoidable. While an ensemble of QDs often results in inhomogeneous broadening and limits the benefits of using QDs in larger laser cavities, the emission linewidth of a single QD is subject to only homogeneous broadening. Thus, at a low temperature, single QD emission within a narrow linewidth nanocavity can result in large enough fields to modify the spontaneous emission processes and to demonstrate strong coupling. The use of narrow-linewidth sources, available from QD emission, is key in providing the desired large enhancement in intensity. In this report, we describe optical properties of 2D photonic crystal slab nanocavities fabricated on $\mathrm{AlO}_{x}$ layers and containing InAs self-assembled QD active material.

Epitaxial QD layers were grown on (001) GaAs by molecular beam epitaxy. Three stacked InAs QD layers were clad by $\mathrm{Al}_{0.16} \mathrm{Ga}_{0.84} \mathrm{As}$ layers on top of a $400 \mathrm{~nm}$ $\mathrm{Al}_{0.94} \mathrm{Ga}_{0.06} \mathrm{As}$ layer. The QDs density in these samples is 3 $\times 10^{10} \mathrm{dots} / \mathrm{cm}^{2}$. A GaAs cap layer is added to protect the top on the final layer. The cavity thickness $(d)$ is designed to be $240 \mathrm{~nm}$. The ground state emission of QDs used in this report showed linewidths as narrow a $43 \mathrm{meV}$.

The patterns to form hexagonal-arrayed photonic crystal defect cavity were defined into a $250 \mathrm{~nm}$ poly(methylmethacrylate) resist spun onto the epilayers and exposed in a field emission electron beam lithography system. Photonic 


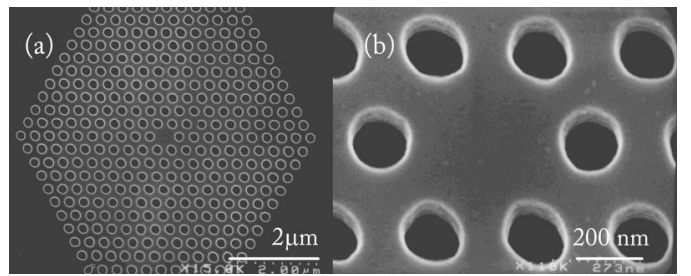

FIG. 1. Images taken by scanning electron microscopy for $2 \mathrm{D}$ photonic crystal single defect cavity used in this work. (a) entire cavity and (b) zoomed around a single defect.

crystal cavities are surrounded by ten layers of PBG, and the lattice spacing $(a)$ is lithographically controlled from 270 to $390 \mathrm{~nm}$. The ratio of hole radius $(r)$ to lattice spacing $(a)$ is similarly tuned from 0.16 to 0.4 . After lithography, the beamwritten patterns were transferred through the active membrane by using an $\mathrm{Ar}^{+}$ion beam assisted with a $\mathrm{Cl}_{2}$ jet, and the $\mathrm{Al}_{0.94} \mathrm{Ga}_{0.06} \mathrm{As}$ layer under cavities was subsequently oxidized in steam at $400{ }^{\circ} \mathrm{C}$ for 5 min to define a perforated dielectric slab structure on top of an $\mathrm{AlO}_{x}$ cladding layer. For most of the structures, the $\mathrm{Al}_{0.94} \mathrm{Ga}_{0.06} \mathrm{As}$ was oxidized as far as $3 \mu \mathrm{m}$ from the edge of photonic crystal hexagons. Figure 1 shows images taken by scanning electron microscopy for typical fully processed photonic crystal cavities with a single defect used in this report. The precise transferred pattern can be seen in Fig. 1. Light parallel to the surface is confined by the Bragg reflection from the photonic crystal in the plane whereas the high index slab structure is used to vertically confine light by total internal reflection.

Optical pumping normal to the surface was carried out by using a $830 \mathrm{~nm}$ semiconductor laser diode with $2.5 \mu \mathrm{s}$ wide pulses and a $3 \mu$ s period. The $830 \mathrm{~nm}$ pump light can be absorbed only by the InAs QDs and the wetting layers, and the pump light could be focused onto the sample with a spot diameter of $2 \mu \mathrm{m}$. Light emission extracted from the surface was then detected by an optical spectrum analyzer. The peak pumping power was $2.4 \mathrm{~mW}$, which corresponds to a power density of $75 \mathrm{~kW} / \mathrm{cm}^{2}$. All of the measurements were performed at room temperature. Figure 2 shows a typical photoluminescence spectrum for photonic crystal single defect cavities with different lattice spacings and the same $r / a$. The cavity resonance was measured to exhibit a 3 to 5 $\mathrm{nm}$ emission linewidth, and the spectrum was found to change significantly by the introduction of small lithographic

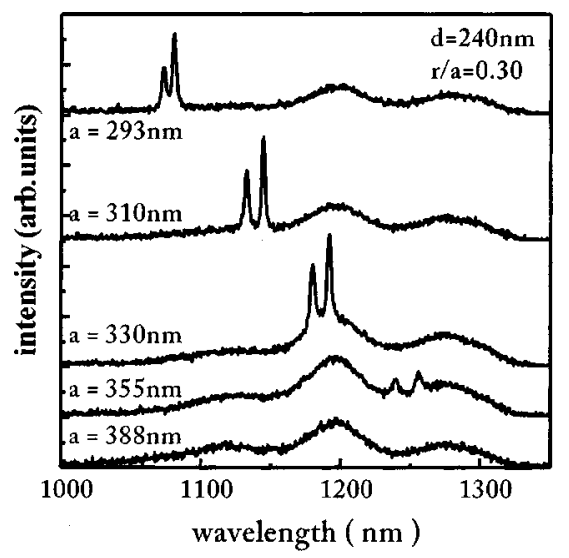

FIG. 2. Photoluminescence spectra for samples with different lattice spacing (a) and same $r / a$ of 0.30 . which favored extended modes. As an example, Fig. 4 shows
Downloaded 18 Dec 2005 to 131.215.240.9. Redistribution subject to AlP license or copyright, see http://apl.aip.org/apl/copyright.jsp

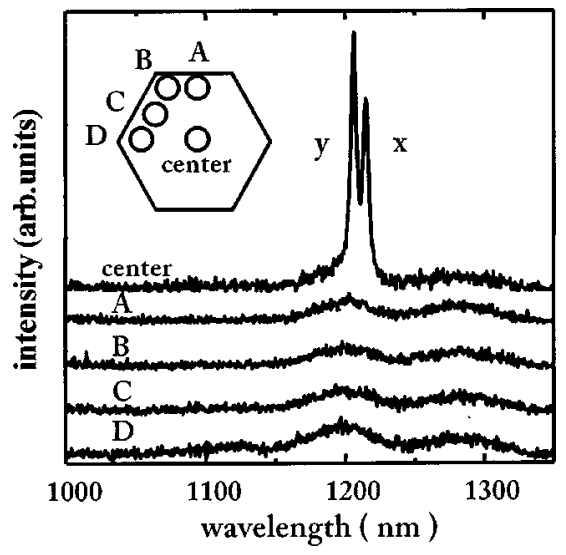

FIG. 3. Position-dependent photoluminescence for a photonic crystal with a single defect. The spectra labeled as A, B, C, and D were taken in each point in a cavity away a single defect as shown in an inset.

changes to the patterns. The spectra of the resonant modes can be tuned throughout the emission range of the QDs by changing the lattice period of the photonic crystal.

We also conducted position-dependent $\mu$-photoluminescence measurement to determine the mode volume of the donor modes. Figure 3 shows photoluminescence spectra taken in different points of the photonic crystal pattern described in the inset. Points labeled as A, B, C, and D are within the photonic crystal, but close to the edge of the pattern. We found that only the spectrum taken from the center of the photonic crystal pattern exhibits the two resonance peaks associated with the optical nanocavity modes while the other points exhibit a much broader QD emission. Although only four points close to the edge of the hexagon are shown in Fig. 3, many other points were tested and this result was carefully verified on many patterns. Optical pumping of points close to the edge was not related to the two distinct peaks in the spectrum which we expect to originate from the localized donor modes. Since the pumping diameter is $2 \mu \mathrm{m}$ and the distance from the center of cavity to the edge is 3.5 $\mu \mathrm{m}$, this indicates that the measured modes are well confined to the single defect cavity. The pair of modes observed in Fig. 3 results from two perpendicular dipole modes in this cavity. When filtering the spectrum with a broad band polarizer, we observe that each of these peaks is purely $x$ or $y$ polarized as described in Fig. 3, and the higher energy line corresponds to $y$-polarized light. Spectral splitting of donor modes ideally does not occur in a completely symmetric single defect cavity within a 2D photonic crystal, since the structure with a $C_{6 v}$ symmetry leaves two degenerate eigenmodes. ${ }^{15}$ Therefore, we expect the measured mode splitting of around 1\% relative to the frequency in Fig. 3 to result from fabrication fluctuations within the photonic crystal close to the cavity. It should be noticed that resonances at $a=355 \mathrm{~nm}$ show broader emission linewidth. This might be a result of many-body effects from the almost fullypopulated ground state of the QDs. Linewidth broadening could also result from changes in the effective photonic bandedge during lithographic tuning since the $d / a$ ratio is inadvertently altered. ${ }^{16}$

We also measured photoluminescence from cavities defined within photonic crystals with different $r / a$ values, 


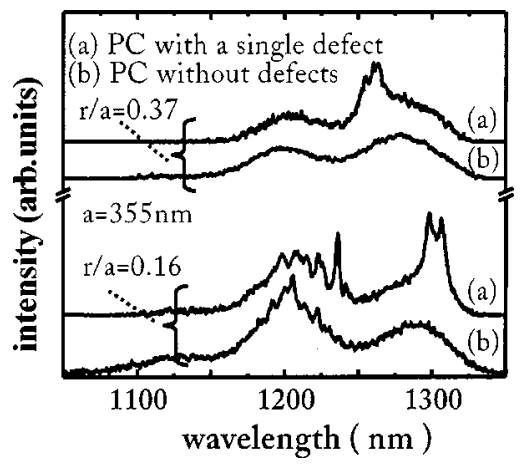

FIG. 4. Photoluminescence spectra for a photonic crystal cavity with a single defect and without defects for $r / a$ of 0.16 and 0.37 . The lattice spacing is kept at $355 \mathrm{~nm}$.

photoluminescence spectra for two photonic crystals with $r / a$ values varying from 0.16 and 0.37 . Photoluminescence spectra from such photonic crystals with single defect cavities can be compared with those taken from photonic crystals without defect cavities. For $r / a=0.37$, the spectrum from the photonic crystal defect cavity again shows the two dipole modes associated with the localized donor mode. There are no other significant features in this spectrum. However, for photonic crystals with $r / a=0.16$, a set of sharp lines are observed in addition to the two dipole resonances. Moreover, the photoluminescence spectra from photonic crystals without defect cavities also demonstrate similar sharp resonances, and these lines are attributed to shallow modes from lithographic variations in the photonic crystal itself. There tends to be a qualitative correlation between the number of the sharp lines and the fluctuations in the hole dimensions from the PBG crystal surrounding the cavities. This discussion is supported by position-dependent $\mu$-photoluminescence spectrum measurements, as shown in Fig. 5, which were taken both in the center and close to edges. Thus, the sharp peaks are signatures of the band edge modes and/or shallow impurity modes. For $r / a$ of 0.16 , the donor mode is closer to the air band edge than ones for $r / a$ of 0.37 , and more likely to support shallow acceptor modes. Thus, we typically do not see both donor modes and shallow band edge modes in the measured range of wavelength.

In summary, we demonstrated InAs QD emission from a 2D photonic crystal nanocavity, which we believe will be essential to large enhancements in light emission. Strongly localized deep donor modes were confirmed to have small mode volumes, verified with position-dependent $\mu$ photoluminescence. The $x$ - and $y$-polarized donor modes could be lithographically tuned over the QD emission range, and the $Q$ 's were measured to be as high as 400 . Extended modes with $Q$ values of up to 2000 were also measured, and are expected to result from small variations of the hole sizes defining the photonic crystals. The observations presented here lead us to believe that, with optimized cavity designs, it

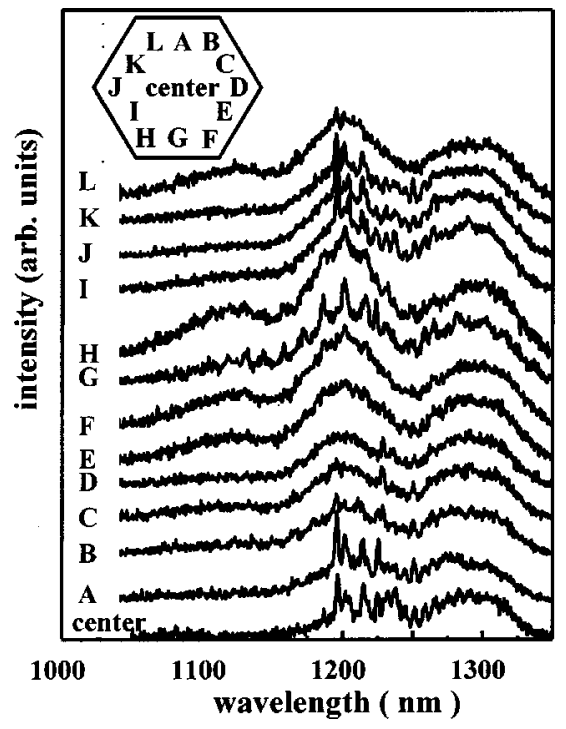

FIG. 5. Position-dependent photoluminescence for a photonic crystal without defects. The spectrum labeled from A to $J$ were taken in each point in a cavity away a single defect as shown in the inset.

is possible to construct a QD photonic crystal laser and to demonstrate strong coupling.

The authors gratefully appreciate many fruitful suggestions from O. Painter, J. Vućkovič, and Marko Lǒnkar. This work was supported by the National Science Foundation, the Army Research Organization, and the Air Force Office of Scientific Research.

${ }^{1}$ E. M. Purcell, Phys. Rev. 69, 681 (1946).

${ }^{2}$ A. Scherer, J. L. Jewell, Y. H. Lee, J. P. Harbison, and L. T. Florez, Appl. Phys. Lett. 55, 2724 (1989).

${ }^{3}$ J. M. Gerard, D. Barrier, J. Y. Marzin, R. Kuszelewicz, L. Manin, E. Costard, V. Thierry-Mieg, and T. Rivera, Appl. Phys. Lett. 69, 449 (1996).

${ }^{4}$ A. F. J. Levi, R. E. Slusher, S. L. McCall, T. Tanbun-Ek, D. L. Coblentz, and S. J. Pearton, Electron. Lett. 28, 1010 (1992).

${ }^{5}$ D. G. Deppe, L. A. Graham, and D. L. Huffaker, IEEE J. Quantum Electron. 35, 1502 (1999).

${ }^{6}$ E. Yablonovitch, T. J. Gmitter, R. D. Meade, A. M. Rappe, K. D. Brommer, and J. D. Joannopoulos, Phys. Rev. Lett. 67, 3380 (1991).

${ }^{7}$ R. D. Meade, A. Devenyi, J. D. Joannopoulos, O. L. Alerhand, D. A. Smith, and K. Kash, J. Appl. Phys. 75, 4753 (1994).

${ }^{8}$ O. Painter, J. Vućkovič, and A. Scherer, J. Opt. Soc. Am. B 16, 275 (1999).

${ }^{9}$ O. J. Painter, R. K. Lee, A. Scherer, A. Yariv, J. D. O’Brien, P. D. Dapkus, and I. Kim, Science 284, 1819 (1999).

${ }^{10}$ J. Hwang, H. Ryu, D. Song, I. Han, H. Song, H. Park, Y. Lee, and D. Jang, Appl. Phys. Lett. 76, 2982 (2000).

${ }^{11}$ W. D. Zhou, J. Sabarinathan, B. Kochman, E. Berg, O. Qasaimeh, S. Pang, and P. Bhattacharya, Electron. Lett. 36, 1541 (2000).

${ }^{12}$ D. Bimberg, M. Grundmann, and M. N. Ledentsov, Quantum Dot Heterostructures (Wiley, New York, 1999).

${ }^{13}$ M. Sugawara, Self-Assembled InGaAs/GaAs Quantum Dots, Semiconductors and Semimetals (Academic, San Diego, 1999), Vol. 60.

${ }^{14}$ M. Boroditsky, I. Gontijo, M. Jackson, R. Vrijen, E. Yablonovitch, T. Krauss, C. C. Cheng, A. Scherer, R. Bhat, and M. Krames, J. Appl. Phys. 87, 3497 (2000).

${ }^{15}$ K. Sakoda, Phys. Rev. B 52, 7982 (1995).

${ }^{16}$ S. G. Johnson, S. Fan, P. R. Villeneuve, and J. D. Joannopoulos, Phys. Rev. B 60, 5751 (1999). 